\title{
USING GENERATIVE ADVERSARIAL NETWORKS FOR EXTRACTION OF INSAR SIGNALS FROM LARGE-SCALE SENTINEL-1 INTERFEROGRAMS BY IMPROVING TROPOSPHERIC NOISE CORRECTION
}

\author{
B. Ghosh ${ }^{1 *}$ M. Haghshenas Haghighi ${ }^{2}$, M. Motagh ${ }^{1,2}$, S. Maghsudi ${ }^{3}$ \\ ${ }^{1}$ GFZ German Research Centre for Geosciences, Department of Geodesy, Section of Remote Sensing, 14473 Potsdam, Germany \\ ${ }^{2}$ Institut für Photogrammetrie und GeoInformation (IPI), Leibniz Universität Hannover, Nienburger Str. 1, D-30167 Hannover \\ ${ }^{3}$ Computer Science Department, Maria-von-Linden-Straße 6, 2nd floor, room no. 20-5 / A19, University of Tübingen, 720740 Tuebingen
}

KEY WORDS: Sentinel-1, Interferometric Synthetic Aperture Radar (InSAR), ERA-Interim, GACOS, Generative Adversarial Network (GAN)

\begin{abstract}
:
Spatiotemporal variations of pressure, temperature, water vapour content in the atmosphere lead to significant delays in interferometric synthetic aperture radar (InSAR) measurements of deformations in the ground. One of the key challenges in increasing the accuracy of ground deformation measurements using InSAR is to produce robust estimates of the tropospheric delay. Tropospheric models like ERA-Interim can be used to estimate the total tropospheric delay in interferograms in remote areas. The problem with using ERA-Interim model for interferogram correction is that after the tropospheric correction, there are still some residuals left in the interferograms, which can be mainly attributed to turbulent troposphere. In this study, we propose a Generative Adversarial Network (GAN) based approach to mitigate the phase delay caused by troposphere. In this method, we implement a noise to noise model, where the network is trained only with the interferograms corrupted by tropospheric noise. We applied the technique over 116 large scale $800 \mathrm{~km}$ long interfergrams formed from Sentinel-1 acquisitions covering a period from 25th October, 2014 to 2 nd November, 2017 from descending track numbered 108 over Iran. Our approach reduces the root mean square of the phase values of the interferogram $64 \%$ compared to those of the original interferogram and by $55 \%$ in comparison to the corresponding ERA-Interim corrected version.
\end{abstract}

\section{INTRODUCTION}

Over the years, various satellites like ERS-1, ERS-2 and Envisat has been in use for the interferometric capability for a wide range of geophysical (Hooper et al., 2007), (Haghshenas Haghighi and Motagh, 2016), (Motagh et al., 2017), engineering (Fornaro et al., 2013) and environmental ((Castel et al., 2000)) applications. Utilizing these Synthetic Aperture Radar (SAR) acquisitions, repeated approximately from the same point in space at different times, Interferometric SAR (InSAR) gives us the differences in path length in the scale of the carrier wavelength, due to changes in wavelength (Massonnet and Feigl, 1998), (Bürgmann et al., 2008). While conventional InSAR suffers from interference from unwanted signals like variations of scattering properties of the earth's surface or atmospheric conditions through time (Hooper et al., 2012), multi-temporal interferometric methods (MTI) including Persistent Scatter InSAR (PSI) (Ferretti et al., 2001), (Hooper et al., 2004) and Small Baseline Subset (SBAS) ((Berardino et al., 2002)) present a specific class of processing that exploits multiple SAR images acquired over an area to separate the displacement signal from the unwanted noise.

While the idea of displacement monitoring in wide area using advanced InSAR time series analysis methods has attracted a great deal of attention in recent years, one of the major limiting factors this suffered from was the non-availability of both spatially and temporally homogeneous SAR data-set in a na-

\footnotetext{
* Corresponding author
}

tionwide or continental scale (Haghshenas Haghighi and Motagh, 2017). With the launches of Sentinel-1A and 1B satellites in 2014 and 2016 respectively, the availability of SAR data from every part of the world has been increased many folds. With short revisit times of 1-6 days, the Sentinel-1 and the planned Tandem-L and NISAR missions provide an unprecedented wealth of topography and surface change data using InSAR technique. With both Sentinel-1A and Sentinel-1B in orbit, this mission currently provides more than 10 TB (Sentinel Data Access Annual Report 2017 - News - Sentinel Online, n.d.) of products every day. Based on its acquisition plans, SAR images from the same orbit are acquired every 6 days over Europe as well as some hotspots with very rapid changes like Greenland (Haghshenas Haghighi and Motagh, 2017). In other parts of the world, SAR data are acquired every 12 or 24 days.

The interferometric phase is affected by differences in propagation delays through troposphere or ionosphere in the time of SAR image acquisitions. The major atmospheric contribution in S-1 interferograms in mid-latitudes comes from the troposphere. This effect is driven by the changes in refractivity of the troposphere at the time of two SAR acquisitions (Haghshenas Haghighi and Motagh, 2017). Two different categories of atmospheric corrections are typically applied on SAR interferograms. In the first category, the atmospheric effect is calculated and mitigated solely based on the phase information of the interferograms. For example, assuming the tropospheric effect behaves randomly in time, it is possible to average several interferograms from the same area to reduce the effect (Zebker et al., 1997). The other category of atmospheric mitigation meth- 
ods is based on external information, in which different sources, such as global atmospheric models, Global Navigation Satellite System (GNSS), or ERA-Interim data that provide information about the atmospheric condition at the time of SAR acquisition, are used for tropospheric correction of interferograms. (Bekaert et al., 2015).

One of the main problems while measuring surface deformation using InSAR is to correct the interferogram from the tropospheric phase delay. To make this correction, the interferometric phase is compared to the atmospheric delays derived from the ERA-Interim global meteorological model and the Global Navigation Satellite System (GNSS).

The ERA-Interim is a global atmospheric model calculated by the European Center for Medium-Range Weather Forecast (ECMWF) based on the assimilation of different input data-sets. It provides several meteorological parameters, including pressure, temperature, and relative humidity at 6-hourly intervals at a grid of $70 \mathrm{~km}$ spatial resolution and 37 vertical intervals from sea level up to $50 \mathrm{~km}$ (Dee et al., 2011). Atmospheric parameters provided by ERA-Interim are interpolated using a vertical and horizontal spline interpolation and a linear interpolation in time to find the atmospheric phase delay from differences in water vapor (wet delay) and atmospheric pressure (hydrostatic delay) at each pixel of the interferogram (Haghshenas Haghighi and Motagh, 2017). The estimated phase delay known as the zenith total delay (ZTD) can then be used to correct the interferogram (Bekaert et al., 2015). From GNSS stations also, wet delay maps with a specific spatial resolution and time interval can be estimated. Tropospheric wet delay data derived from GNSS can be successfully used to correct InSAR displacement maps ( $\mathrm{Li}$ et al., 2005). However, in remote areas or countries with very less GNSS stations, correction by the ERA-Interim model becomes the only choice. The problem with using ERA-Interim model for interferogram correction is that after the tropospheric correction, there are still some residuals left in the interferograms, which can be mainly attributed to turbulent troposphere. This is due to the spatial resolution of the atmospheric models by ERA-Interim, which are coarser than the resolution of the S-1 interferograms. Therefore, they are not able to completely remove the turbulent tropospheric effect from the interferograms (Jolivet et al., 2011), (Haghshenas Haghighi and Motagh, 2017).

\subsection{Our Contribution}

One method to extract the features from the interferograms corrupted by noise and to remove the turbulence noise from these interferograms is to implement a convolutional neural network which can learn and generate the corrected version of the interferogram free from external noise. The papers (Bermudez et al., 2018), (Enomoto et al., 2017) and (Grohnfeldi et al., 2018) describe the use of Generative Adverserial Networks (GAN) for SAR image translation. There exists quite a lot of work on denoising of images as well as using Gaussian Processes (GP) based approach for improving tropospheric correction of large scale interferograms (Shamshiri et al., 2020). However, we found that deep learning has not been applied in literature for removing noise from interferograms so far. The existing methods for noise removal from interferograms mainly involve stacking of the interferograms and extraction of the average values, which removes noise to quite some extent.

GANs have been used quite a lot for deblurring and denoising of images like DeblurGAN (Kupyn et al., 2017) uses conditional GANs to deblur images. But there are no documented attempts for implementation of GANs for noise reduction in interferograms.

In this paper, we have applied a variation of the Pix2pix (Isola et al., 2016) algorithm for removing tropospheric noise from the interferograms. For our dataset of choice, we have focused on Iran and used the unwrapped differential phase derived by large scale interferograms of Sentinel-1 and the differential ZTDs derived from the ERA-Interim model at the respective acquisition times as the interferograms. We have implemented a Generative Adverserial Network on the interferometric phase post removal of ZTDs from the ERA-Interim model to further remove the residual turbulent noise.

The rest of the paper is organized as follows. Section 2 provides an overview of the theoretical background. It also introduces the proposed method by detailing both the used architecture and the adopted learning strategy. In Section 4, we describe the suite of experiments, accurately designed to validate our approach, together with the datasets generation process and the analysis of the obtained results. In Section 5, we derive our results for our experiments. Finally, we derive our conclusions and outline possible future work in Section 7.

\section{RELATED WORK}

The development of powerful computing devices and increasing availability of large amount of data, have led to the deployment of machine learning methods, which are able to learn from the existing data. The performance of these methods in complex tasks like image classification (Redmon and Farhadi, 2016) and object detection (Simonyan and Zisserman, 2014) are quite noteworthy. In remote sensing, Deep Learning methods have been proven to be quite capable because of their ability to automatically learn suitable features from images, without manually setting the parameters of specific algorithms (Li et al., 2018). In recent years, the use of Deep Learning has also been investigated for image denoising tasks. Many previous solutions (Pathak et al., 2016), (Wang and Gupta, 2016) to problems in this area have used an encoder-decoder network (Hinton and Salakhutdinov, 2006). Such networks are neural networks consisting of a neural encoder network that maps high dimensional samples of an unknown distribution to a lower-dimensional representation, and a decoder that reconstructs the input from that compressed representation. Applications of these networks involve outlier detection, or dimensionality reduction for further processing. However such networks cannot be applied to generate realistic samples (Lee and Lee, 2018). One reason for this is that only a tiny fraction of the encoding space is used by the encoder-decoder network to encode realistic samples. In an encoder-decoder network, most of the low-level information shared between the input and output is shunted because of the bottle-neck of passing through all the layers. One defining feature of using a GAN architecture is that they map a high resolution input grid to a high resolution output grid. In this paper, we applied a GAN architecture which takes an interferogram, corrupted by tropospheric noise, as input and then train the network to remove the noise to some extent.

Generative Adversarial Networks (GANs) are very popular since the last years for learning how to sample from complex high dimensional distributions $P_{X}: \mathcal{X} \mapsto[0,1]$ such as images of faces (Gross, 2005). It is achieved by training a generator $G: \ddagger \mapsto \mathcal{X}$ to map samples from a prior $P_{z}: \ddagger \mapsto[0,1]$ in a so-called latent space to images and training a discriminator 
$D: \mathcal{X} \mapsto[0,1]$ to distinguish these fakes from real samples The GAN follows an adversarial procedure. The discriminator classifies real samples from generated ones (fakes), while the generator learns to fool the discriminator. Since the introduction of the original GAN by Goodfellow et al (Goodfellow et al., 2014), various architectures and applications have been explored.

\section{METHODOLOGY}

Our method for reduction of noise from interferograms involves the following steps:

1. The interferograms are first corrected for tropospheric delay using the Zenith Total Delay (ZTD) map derived from the ERA-Interim database.

2. To reduce computation power, the corrected interferograms are divided into patches.

3. All the patches from the interferograms are stacked together.

4. A fraction of total number of patches extracted are then used to train the GAN model.

5. Once the model has been trained, noise corrected patches of the remaining interferograms, not used in training, are then synthesized by the generator unit.

6. The predicted patches are concatenated to form the full interferograms with the noise removed.

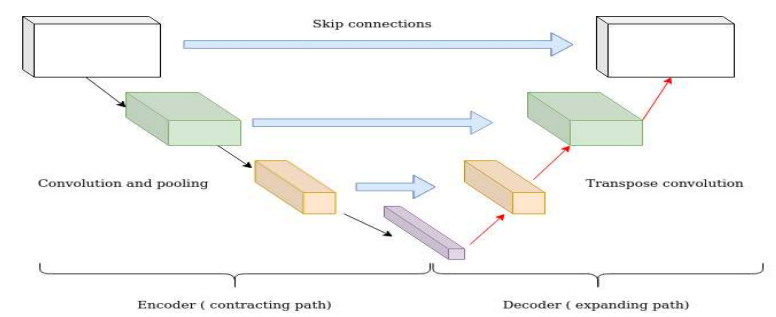

Figure 1. Generator with skip connections (Adaloglou, 2020).

The generator takes the interferogram with the phase value corrupted by tropospheric noise as input and provides the predicted interferogram with the noise reduced.By introducing skip connections in the encoder-decoder architecture of the generator, fine-grained details can be recovered in the prediction.

For our work, the generator architecture (Figure 1) follows the general shape of a "U-Net" (Ronneberger et al., 2015). Similar to the architecture proposed in (Isola et al., 2016), skip connections are added so that low-level information does not have to pass through all the layers and thus circumvent a bottle-neck situation. Specifically, skip connections are added between each layer $i$ and layer $n-i$, where $n$ is the total number of layers.

For the discriminator, a patch-based architecture is applied, which tries to classify if each $N \times N$ patch in an image is real or fake. This discriminator is run convolutionally across the image, averaging all responses to provide the final output of the discriminator (Isola et al., 2016)).

Usually for reduction of noise from images or any other objects, the aim is to recover a clean image $x$ from the observed image $y$, corrupted by noise, such that $y=x+$ noise (Xu et al., 2019). Usually the noise is the additive white Gaussian noise (AWGN). But, in real life situations, such as working with interferograms, corrupted by tropospheric noise, it is very difficult to simulate the statistical distribution of tropospheric noise, for supervised learning, as this distribution is very different from additive white Gaussian noise (AWGN). One more factor is that it is impossible to acquire fully clean interferograms, devoid of tropospheric noise, for supervised learning purposes.

The paper (Lehtinen et al., 2018) proposes a method to overcome this problem, by observing that it is possible to learn to transform noisy images into clean images by only looking at noisy images. The results as presented in (Lehtinen et al., 2018) claims that this model may work even better than supervised learning with clean and noisy image pairs.

For our work as well, we follow a similar approach. Since, we only have the interferograms with troposheric noise, and no corresponding noise-free version of the interferograms are available, we train both the generator as well the discriminator on the same input, which are the interferograms corrupted with troposheric noise. The advantage of this method is that we neither require an explicit statistical likelihood model of the noise nor a noise-free prior model of the intefergram, instead learn these indirectly from the training data.

\section{EXPERIMENTAL PROCEDURE}

\subsection{Datasets}

For this study, 52 Single Look Complex (SLC) images were acquired from the Sentinel-1 in IW mode, covering dates from 25th October, 2014 to 2nd November, 2017 from descending track numbered 108 over Iran, timed at 02.45 .52 hours. 116 interferograms were processed using the Gamma software (Sensing, n.d.). The interferograms were formed with a temporal baseline of 180 days on average, taking the earlier date as master and the later date as slave. After merging consecutive frames of the Sentinel-1 to produce a large SLC image and applying precise orbital data, it was possible to generate a largescale interferogram covering $800 \mathrm{~km}$. The orbital and topographic phases were removed by precise orbit data and SRTM 90-meter Digital Elevation Model (DEM) (Farr and Kobrick, 2000), (SRTM 90m Digital Elevation Database v4.1, 2017). The interferograms were then geo-coded and unwrapped.

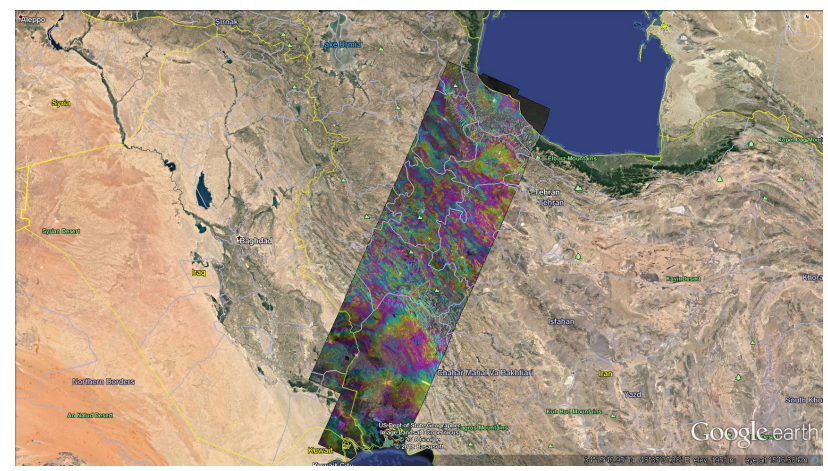

Figure 2. Interferograms were formed from Sentinel-1 SAR acquisitions along the descending track 108 superimposed on the aerial map from Google Earth (Michelson, 2017), covering dates from 25 October, 2014 to 2nd November, 2017. 


\subsection{Applying atmospheric delay correction by ERA-Interim} model

As mentioned earlier, ERA-Interim provides pressure, temperature, and relative humidity along with other meteorological data. The ZTD, using these variables, is calculated for each node at the SAR acquisition time. This data is downloaded for the corresponding interferogram dates from the Generic Atmospheric Correction Online Service for InSAR (GACOS) (Yu et al., 2018b), (Yu et al., 2018a), (Yu et al., 2017). GACOS utilises the Iterative Tropospheric Decomposition (ITD) model (Yu et al., 2017) to separate stratified and turbulent signals from tropospheric total delays, and generate high spatial resolution zenith total delay (ZTD) maps to be used for correcting InSAR measurements. The ZTD maps were acquired from the GACOS website for the respective dates. The tropospheric phase delay of the intereferograms were estimated by subtracting the ZTD maps from the original phase of the interferograms. To account for residual orbital errors, the original interferograms were corrected from a linear trend in range.
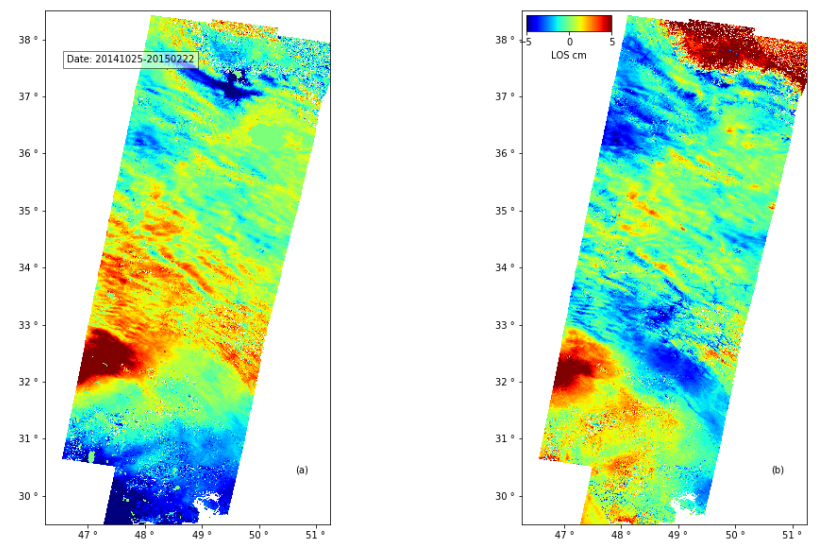

Figure 3. Full-length interferograms are plotted for the dates 20141025-20150129 in (a). The corresponding tropospheric delay was predicted using ERA-Interim model in (b)

\subsection{Generation of binary mask to hide deformation areas during training}

Our aim was to train the model, so that it can learn to reduce the tropospheric noise. To make the model train on only the residual tropospheric noise, and not on deformation and subsidence data, the deformation areas had to be masked. This was achieved by stacking and averaging all the interferograms together and this helped in identifying the deformation or subsidence zones on the interferogram. A binary mask (figure 4) was generated which converted all the pixels at the deformation areas to zero. This binary mask was then applied to all the interferograms to completely hide the deformation areas while training.

\subsection{Generating training and testing datasets}

As mentioned in the paper (Yu et al., 2018b), there are several performance indicators which give an overview regarding the accuracy of the ZTD maps. One such performance indicator is the correlation between the original interferometric phase and the estimated tropospheric delay from ERA-Interim. A high correlation between phase measurements and the computed atmospheric corrections suggests that the model is able to capture
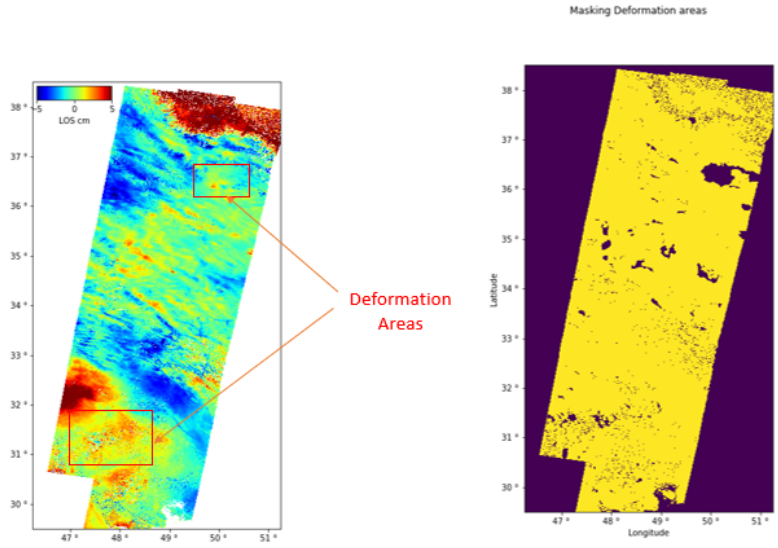

Figure 4. The deformation areas were identified using stacking from the original interferograms in a). A binary mask generated from the all the interferograms with the deformation areas hidden as shown in b). This binary mask was used to hide the deformation areas in all the interferograms during training the model.

most of the atmospheric effects, and thus successful InSAR atmospheric error correction is expected.

For all 116 interferograms, the correlations between the interferometric phase and tropospheric delays per pixel were calculated. For our training set, we selected 91 of the 116 interferograms having correlation of more than $60 \%$ as our training set and the rest for validation and testing.

\subsection{Training}

The size of each interferogram was 9472 by 5120 pixels. To reduce computation power and training time, we decided to train the model on patches of 256 by 256 pixels, from all the interferograms of the training set. Thus, all the 91 interferograms were then divided into patches of 256 by 256 pixels. All the patches were then stacked together and 6000 of them were randomly selected and used for training the model. As mentioned in ??, we train the model by using the same input of the interferogram patches, into both the generator as well as the discriminator of the GAN. With every epoch of training, the generator generates a new version of the input interferogram patch with reduced noise, and the discriminator tries to distinguish between the generated patch from the discriminator and the original input.

We use the same training procedure as adopted for the paper (Isola et al., 2016). We use Tensorflow (Abadi et al., 2015) with Keras (Chollet et al., 2015), with Google colab (Colaboratory, n.d.) as the platform. The hyperparameters of the model are listed in Table 1. Mean square loss is used to train the network as this loss is more stable. We use Adam optimizer with learning rate of 0.0002 and momentum of 0.5 for training the GAN. The network is trained for 500 epochs for noise removal. We use kernel size of $4 \times 4$ and zeropadding by 1 , with a stride of 2 and 1 , for all convolutional and deconvolutional layers of generator network, respectively. In case of discriminator network, the first four convolutional and deconvolutional layers were composed of kernels of size $4 \times 4$ with a stride 2 and zero-padding by 1 . However, the last layer in discriminator network uses kernel of size $4 \times 4$ with stride of size of 1 . 
Table 1. Hyperparameters used while training the model

\begin{tabular}{|c|l|}
\hline Hyperparameter & Value \\
\hline \hline Loss & $\begin{array}{l}\text { Mean } \\
\text { Loss }\end{array}$ \\
\hline Optimizer & Adam Optimizer \\
\hline Learning Rate & 0.0002 \\
\hline Momentum & 0.5 \\
\hline Kernel size & $4 \times 4$ \\
\hline $\begin{array}{c}\text { Stride for Generator } \\
\text { convolutional layers }\end{array}$ & 2 \\
\hline $\begin{array}{c}\text { Stride for Generator } \\
\text { deconvolutional layers }\end{array}$ & 1 \\
\hline $\begin{array}{c}\text { Stride for } \\
\text { Discriminator } \\
\text { convolutional layers }\end{array}$ & 2 \\
\hline $\begin{array}{c}\text { Stride for } \\
\text { Discriminator } \\
\text { deconvolutional layers }\end{array}$ & 2 \\
\hline \hline
\end{tabular}

For feeding data to the network, an input data pipeline was designed which loaded the data into the training model in batches to lower the memory consumption while training. Once the model was trained, it was used to predict the corresponding patches on the test set. For generation of the full length interferogram, the test interferograms were split into patches of 256 by 256 pixels. The model was applied on each 256 by 256 patch, and then after all the patches have been predicted, they were stitched back together to get the entire predicted interferogram.

\section{RESULTS}

The model was trained for 500 epochs after which both the generator and discriminator losses did not decrease further. The accuracy of the generated data as evaluated by the discriminator also increased with epoch. Figure 5 shows the predicted inteferogram patches of the test data by the trained model. Most of the tropospheric noise was removed in the predicted patches.

[a]
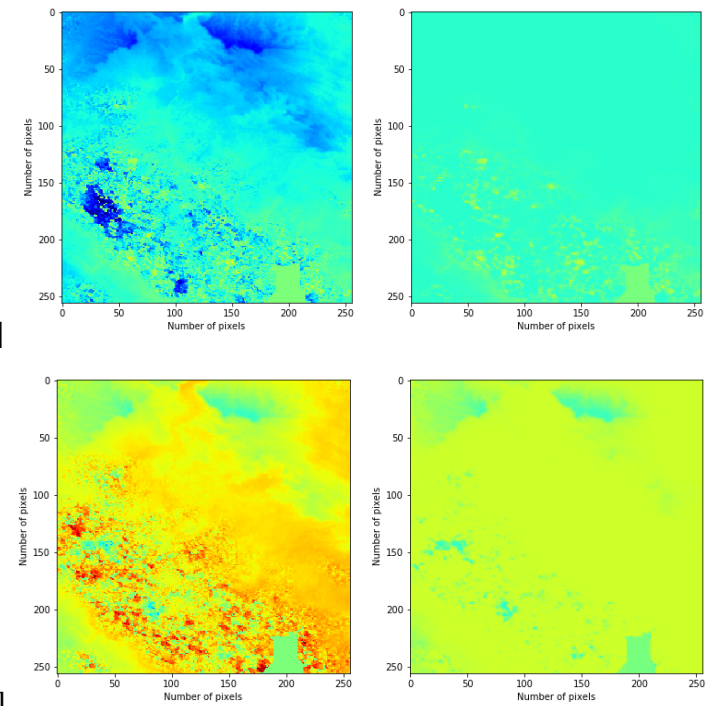

Figure 5. Figures a) and b) showing random patches from test data and corresponding patches after removal of noise using the GAN method

As mentioned in Section (4.5), the model was then used to pre- dict the full length of the interferograms in the test set, as shown in figure 6. In this case also, the tropospheric noise was removed to some extent in the predicted interferograms.
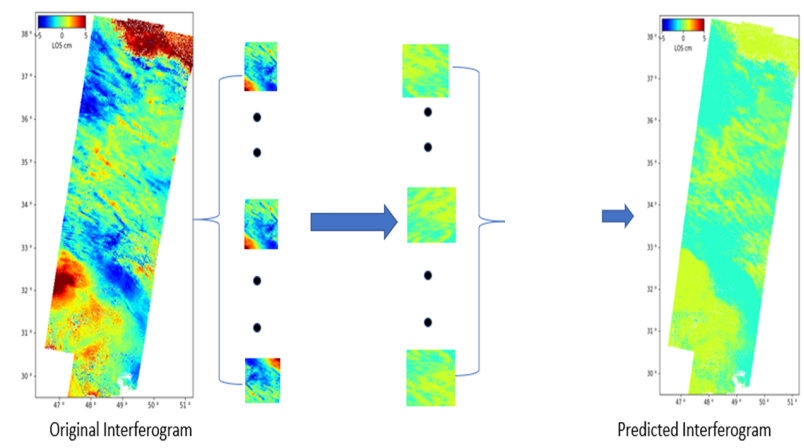

Figure 6. For reducing noise from full-length interferograms, the interferograms were first split into patches of 256 by 256 pixels each, each patch was sent into the trained model as input and resultant patches were then stitched back together to get the full-length interferogram with reduces noise.

\subsection{Comparison of Root Mean Square (RMS) of the phase values}

To evaluate the performance of the model, the root-mean-squared of the phase values (RMS) of the original unwrapped interferograms, the interferograms after correction by the ERA-Interim model and the predicted interfreograms were then calculated and plotted. As shown in figure 7, the RMS values are reduced by $64 \%$ compared to those of the original interferogram and by $55 \%$ in comparison to the corresponding ERA-Interim corrected version. Figure 8 shows the range of the RMS values for the original phase, the corresponding estimated phase delay by the ERA-Interim model, and the predicted phase of the interferogram as generated by our method after noise reduction. It can been seen from the figure that the range of the predicted phase of the interferogram as generated by our method is much lower than the original phase and the ERA-interim estimation.

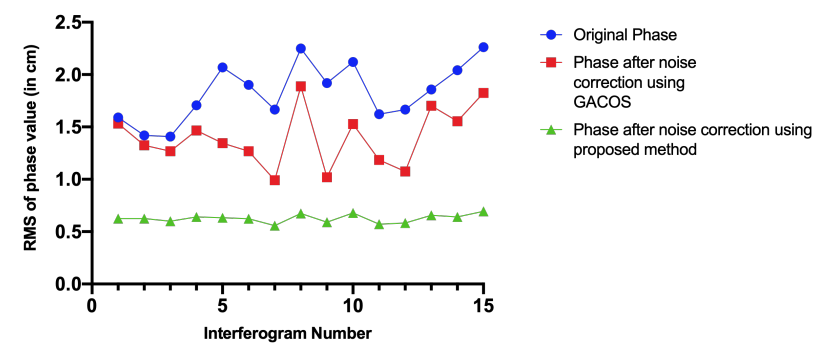

Figure 7. The RMS (Root Mean Square) values were plotted for the original unwrapped phase vs the phase after correction by ERA-Interim (GACOS) in vs the corresponding predicted phase of the interferogram as generated by our method. The RMS values of the predicted phase of the interferogram as generated by our method is much lower compared to the original phase and the ERA-interim estimation.

\section{DISCUSSION}

Our study with Sentinel-1 data showed that ERA-Interim is not able to completely reduce the tropospheric phase delay in large-scale interferometric measurement. Our proposed method based on generative adversarial networks, exploiting $800 \mathrm{~km}$ 


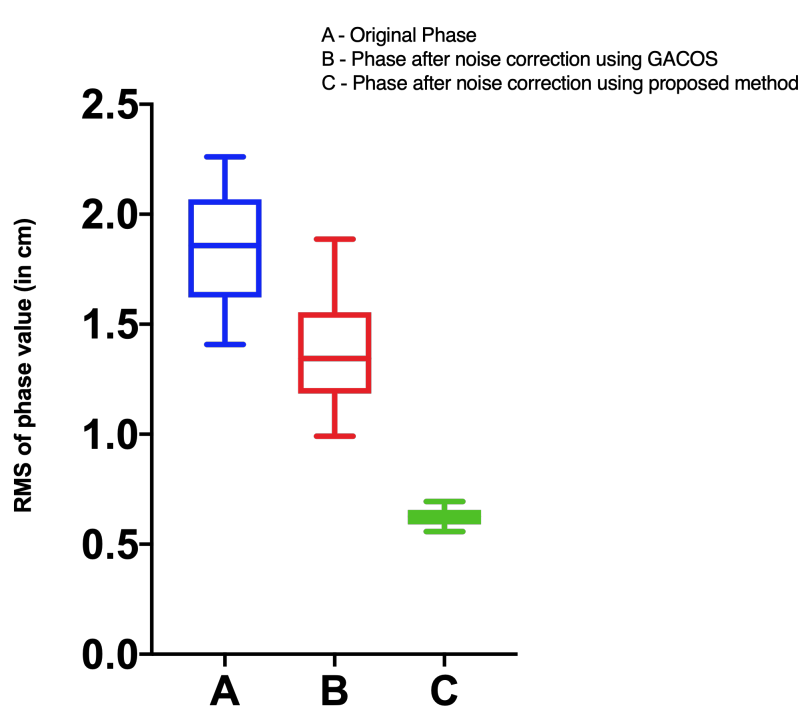

Figure 8. Box-plot denoting the range of the RMS (Root Mean Square) values of the original unwrapped phase vs the phase after correction by ERA-Interim (GACOS) vs the corresponding predicted interferogram. The range of the predicted phase of the interferogram as generated by our method is much lower between 0.5 and 1, than the original phase and the ERA-interim estimation

long intefreograms, improves tropospheric corrections by $55 \%$ compared to the corrections by ERA-Interimmodel. This method is robust and adaptive as it can be also trained on new data and thus capture any changes in the dataset and this will be reflected in the model as well. Also, it has been shown that it is possible to train the model on a fraction of the full dimensions of the interferogram and still facilitate atmosphpheric correction on the entire length and width of the interferogram data, thus proving that this method is also scalable. To further evaluate our proposed method, few additional checks were performed.

\subsection{Comparison of Root Mean Squared (RMS) of the phase values over individual patches across the full length of the interferogram}

For a good correction, the RMS values also need to be reduced uniformly over the whole length of the interferogram. To evaluate our model, we divided the entire inteferogram into 700 random patches and calculated the RMS of each patch. The RMS of each patch was plotted along with the resultant RMS of the corrresponding patch from the original interferogram and the ERA-Interim corrected version. As shown in figure 9, the values in the RMS curve for each of the zones of the predicted interferogram are much lower than the RMS values of the corresponding patches of the other two.

\subsection{Calculating Error-Distance bias as a metric for evalu- ation of noise reduction}

One of the important aspects of tropospheric noise correction of interferograms is to remove any bias of the phase values with distance between the two points at which the phase values are measured. This bias of error with distance should not exist in an ideal interferogram without any noise.

To evaluate if any such bias exists with our proposed method of noise correction, we iteratively calculate the differences in
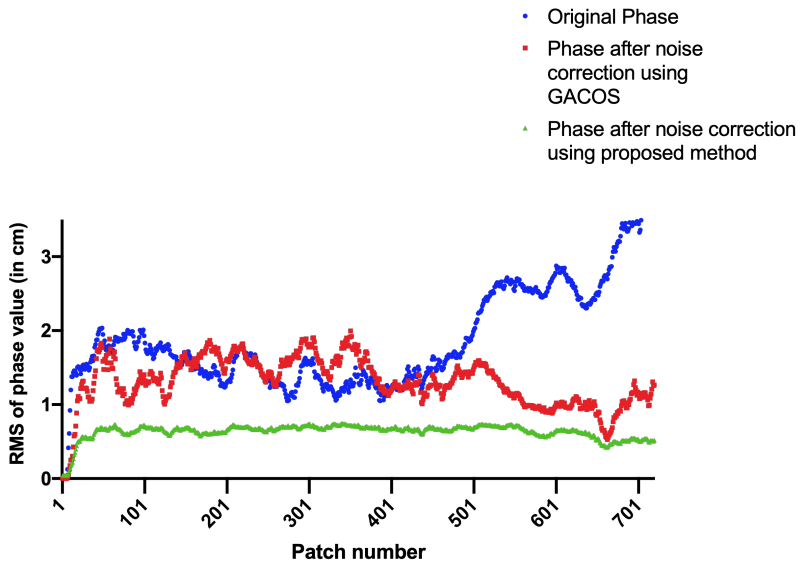

Figure 9. The RMS (Root Mean Square) value changes over the separate patches were calculated and plotted for the original phase vs phase after the correction by ERA-Interim (GACOS) vs the Predicted phase. The values in the RMS curve for each of the patches of the predicted interferogram are much lower than the RMS values of the corresponding patches of the other two.

phase value between random points in the interferogram at specific distances. We calculate the difference in values of the phase in the predicted interfergram, the original interferogram and corresponding ERA-Interim corrected version, at specific distances of 10,20, 30 upto $500 \mathrm{~km}$.

For each distance measured, we randomly sample 1000 pairs of points, calculate the difference in phase value at those points and finally, find the mean and the standard deviation for each distance measured. The mean of the differences were plotted against the distance for the three types of the interferograms. As shown in figure 10, our method reduces the error-distance bias by $53 \%$ in comparison to the original interferogram phase and corresponding ERA-Interim corrected version.

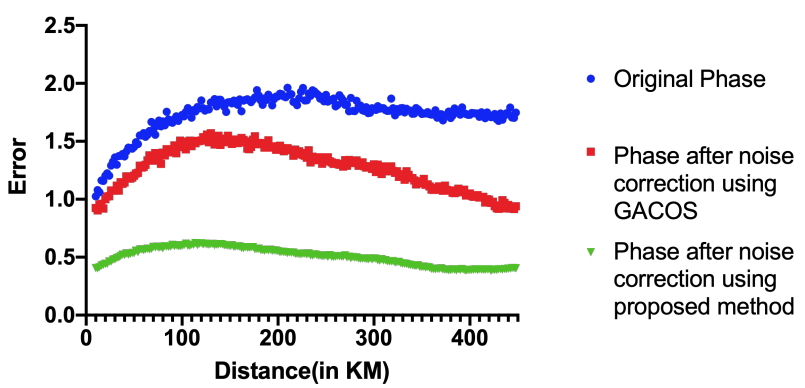

Figure 10. Mean Error vs Distance were calculated and plotted for the original phase vs phase after the correction by

ERA-Interim (GACOS) vs the Predicted phase using the GAN method for Interferogram dated 20141025-20150129. Our method reduces the error-distance bias considerably in comparison to the original interferogram phase and corresponding ERA-Interim corrected version.

\section{CONCLUSION}

In this paper, we presented an adaptation of the Pix2pix GAN model, originally conceived for image to image translation, for the problem of tropospheric noise removal in interferograms. We built an architecture for training the model to generate interferograms with the tropospheric noise reduced by a certain fraction on a large scale interferogram spanning over $800 \mathrm{Km}$, and 
we extensively ran experiments to validate the performance of the proposed approach. Through the experiments, we showed how our model outperforms the tropospheric noise correction method by the ERA-Interim model, as testified by the metrics. Compared to the ERA-Interim method, our approach showed that we are able to reduce most of the tropospheric noise as well as reduce the error-distance bias in the interferograms compared to the ERA-Interim method. We demonstrated how the learned model is effective in filtering out the noise from large scale interferograms by training it only on a certain fraction of the whole interferogram, which saves considerable computational power as well as overhead.

One of the demerits of this method is the considerable training time required to train the model as well as large amount of memory required for the overhead. We processed 116 interferograms and trained the model on 91 of them which lead to considerable overhead during processing and training. Processing the model on the GPU in Google Colab helped to reduce the training time by a substantial amount. But, side by side, and training the entire data-set in Google Drive leads to memory constraint. Having more access to GPUs would have reduced the training time more as well as the problem of memory constraint may also be reduced by training the model in the server. However, we designed an input data pipeline which enabled us to train the model on batches, which reduced the memory overhead to some extent.

\subsection{Future work}

One of the improvements on using a Conditional GAN for removal of noise of interferograms is using a CycleGAN. A CycleGAN has two pairs of generators and discriminators. One pair focuses on converting source domain to target domain while the other pair focuses on the reverse. This bi-directional conversion process allows for a cyclic consistency loss for CycleGAN which ensures the effective conversion from source to target and then back to source again. The transitivity property of cyclicconsistency loss allows CycleGAN to perform well on unpaired translation. CycleGANs are also known to perform very well for background noise reduction in blurry images (Sharma et al., 2019) and so, it may be of interest to see whether the CycleGAN can perform better than the Conditional GAN for noise removal in inteferograms.

\section{REFERENCES}

Abadi, M., Agarwal, A., Barham, P., Brevdo, E., Chen, Z., Citro, C., Corrado, G. S., Davis, A., Dean, J., Devin, M., Ghemawat, S., Goodfellow, I., Harp, A., Irving, G., Isard, M., Jia, Y., Jozefowicz, R., Kaiser, L., Kudlur, M., Levenberg, J., Mané, D., Monga, R., Moore, S., Murray, D., Olah, C., Schuster, M., Shlens, J., Steiner, B., Sutskever, I., Talwar, K., Tucker, P., Vanhoucke, V., Vasudevan, V., Viégas, F., Vinyals, O., Warden, P., Wattenberg, M., Wicke, M., Yu, Y., Zheng, X., 2015. TensorFlow: Large-scale machine learning on heterogeneous systems. Software available from tensorflow.org.

Adaloglou, N., 2020. Intuitive Explanation of Skip Connections in Deep Learning. https://theaisummer.com/. https://theaisummer.com/skip-connections/.

Bekaert, D. P. S., Walters, R. J., Wright, T. J., Hooper, A. J., Parker, D. J., 2015. Statistical comparison of InSAR tropospheric correction techniques. Remote Sensing of Environment, 170, 40-47. http://www.sciencedirect.com/science/article/pii/S0034425715301231.
Berardino, P., Fornaro, G., Lanari, R., Sansoti, E., 2002. A new algorithm for surface deformation monitoring based on small baseline differential SAR interferograms. IEEE Trans. Geosci. Remote Sens. 40, 2375-2383.

Bermudez, J. D., Happ, P. N., Oliveira, D. A. B., Feitosa, R. Q., 2018. SAR TO OPTICAL IMAGE SYNTHESIS FOR CLOUD REMOVAL WITH GENERATIVE ADVERSARIAL NETWORKS. ISPRS Annals of Photogrammetry, Remote Sensing and Spatial Information Sciences, IV-1, 5-11. https://www.isprs-ann-photogramm-remote-sens-spatialinf-sci.net/IV-1/5/2018/

Bürgmann, R., Rosen, P., Fieldling, E., 2008. Synthetic Aperture Radar Interferometry to Measure Earth's Surface Topography and Its Deformation. Annu. Rev. Earth Planet. Sci. 28 , 169-209.

Castel, T., Martinez, J.-M., Beaudoin, A., Wegmüller, U., Strozzi, T., 2000. ERS INSAR Data for Remote Sensing Hilly Forested Areas. Remote Sens. Environ. 73, 73-86.

Chollet, F. et al., 2015. Keras. https://keras.io.

Colaboratory, n.d.

Dee, D. P., Uppala, S. M., Simmons, A. J., Berrisford, P., Poli, P., Kobayashi, S., Andrae, U., Balmaseda, M. A., Balsamo, G., Bauer, P., Bechtold, P., Beljaars, A. C. M., Berg, L. v. d., Bidlot, J., Bormann, N., Delsol, C., Dragani, R., Fuentes, M., Geer, A. J., Haimberger, L., Healy, S. B., Hersbach, H., Hólm, E. V., Isaksen, L., Kållberg, P., Köhler, M., Matricardi, M., McNally, A. P., Monge-Sanz, B. M., Morcrette, J.-J., Park, B.-K., Peubey, C., Rosnay, P. d., Tavolato, C., Thépaut, J.-N., Vitart, F., 2011. The ERA-Interim reanalysis: configuration and performance of the data assimilation system. Quarterly Journal of the Royal Meteorological Society, 137(656), 553-597. https://rmets.onlinelibrary.wiley.com/doi/abs/10.1002/qj.828.

Enomoto, K., Sakurada, K., Wang, W., Fukui, H., Matsuoka, M., Nakamura, R., Kawaguchi, N., 2017. Filmy Cloud Removal on Satellite Imagery with Multispectral Conditional Generative Adversarial Nets. 2017 IEEE Conference on Computer Vision and Pattern Recognition Workshops (CVPRW), 1533-1541. http://arxiv.org/abs/1710.04835. arXiv: 1710.04835.

Farr, T. G., Kobrick, M., 2000. Shuttle radar topography mission produces a wealth of data. Eos, Transactions American Geophysical Union, 81(48), 583-585. https://agupubs.onlinelibrary.wiley.com/doi/abs/10.1029/EO081i$048 \mathrm{p} 00583$.

Ferretti, A., Prati, C., Rocca, F., 2001. Permanent scatterers in SAR interferometry. IEEE Trans. Geosci. Remote Sens. 39, 8 20.

Fornaro, G., Reale, D., Verde, S., 2013. Bridge Thermal Dilation Monitoring With Millimeter Sensitivity via Multidimensional SAR Imaging. IEEE Geosci. Remote Sens. Lett. 10, 677681.

Goodfellow, I. J., Pouget-Abadie, J., Mirza, M., Xu, B., Warde-Farley, D., Ozair, S., Courville, A., Bengio, Y., 2014. Generative Adversarial Networks. arXiv:1406.2661 [cs, stat]. http://arxiv.org/abs/1406.2661. arXiv: 1406.2661.

Grohnfeldi, C., Schmitt, M., Zhu, X., 2018. A Conditional Generative Adversarial Network to Fuse Sar And Multispectral Optical Data For Cloud Removal From Sentinel-2 Images. IGARSS 2018 - 2018 IEEE International Geoscience and Remote Sensing Symposium, IEEE, Valencia, 1726-1729.

Gross, R., 2005. Face Databases. A. S.Li (ed.), Handbook of Face Recognition, Springer, New York.

Haghshenas Haghighi, M., Motagh, M., 2016. Assessment of ground surface displacement in Taihape landslide, New Zealand, with C-and X-band SAR interferometry. N.Z. J. Geol. Geophys., 59, 136-146. 
Haghshenas Haghighi, M., Motagh, M., 2017. Sentinel-1 InSAR over Germany: Large-scale interferometry, atmospheric effects, and ground deformation mapping. ZFV - Zeitschrift fur Geodasie, Geoinformation und Landmanagement 142(4), 245246.

Hinton, G. E., Salakhutdinov, R. R., 2006. Reducing the Dimensionality of Data with Neural Networks. Science, 313(5786), 504-507. https://science.sciencemag.org/content/313/5786/504.

Hooper, A., Bekaert, D., Spaans, K., Ankan, M., 2012. Recent advances in SAR interferometry time series analysis for measuring crustal deformation. Tectonophysics 514-517, 1-13.

Hooper, A., Segall, P., Zebkar, H., 2007. Persistent scatterer interferometric synthetic aperture radar for crustal deformation analysis, with application to Volcán Alcedo, Galápagos. J. Geophys. Res. B: Solid Earth 112.

Hooper, A., Zebkar, H., Segall, P., Kampes, B., 2004. A new method for measuring deformation on volcanoes and other natural terrains using InSAR persistent scatterers. Geophys. Res. Lett. 31

Isola, P., Zhu, J.-Y., Zhou, T., Efros, A. A., 2016. Imageto-Image Translation with Conditional Adversarial Networks. arXiv:1611.07004 [cs]. http://arxiv.org/abs/1611.07004. arXiv: 1611.07004 .

Jolivet, R., Grandin, R., Lasserre, C., Doin, M.-P., Peltzer, G., 2011. Systematic InSAR tropospheric phase delay corrections from global meteorological reanalysis data. Geophysical Research Letters, 38, L17311. https://hal.archives-ouvertes.fr/hal00657439.

Kupyn, O., Budzan, V., Mykhailych, M., Mishkin, D., Matas, J., 2017. DeblurGAN: Blind Motion Deblurring Using Conditional Adversarial Networks. arXiv:1711.07064 [cs]. http://arxiv.org/abs/1711.07064. arXiv: 1711.07064.

Lee, G., Lee, S., 2018. Gan with autoencoder and importance sampling. SIGGRAPH Asia 2018 Posters, SA '18, ACM, New York, NY, USA, 21:1-21:2.

Lehtinen, J., Munkberg, J., Hasselgren, J., Laine, S., Karras, T., Aittala, M., Aila, T., 2018. Noise2Noise: Learning Image Restoration without Clean Data. CoRR, abs/1803.04189. http://arxiv.org/abs/1803.04189.

Li, Y., Zhang, H., Xue, X., Jiang, Y., Shen, Q., 2018. Deep learning for remote sensing image classification: A survey.

Li, Z., Muller, J.-P., Cross, P., Fielding, E. J., 2005. Interferometric synthetic aperture radar (InSAR) atmospheric correction: GPS, Moderate Resolution Imaging Spectroradiometer (MODIS), and InSAR integration. Journal of Geophysical Research: Solid Earth, 110(B3). https://agupubs.onlinelibrary.wiley.com/doi/abs/10.1029/2004JB 003446.

Massonnet, D., Feigl, K., 1998. Radar interferometry and its application to changes in the Earth's surface. Rev. Geophys., 441-500.

Michelson, D., 2017. Google Earth 2017: Learning the Essentials. 1st edn, CreateSpace Independent Publishing Platform, USA.

Motagh, M., Shamshiri, R., Haghshenas Haghighi, M., Wetzel, H.-U., Akbari, B., Nahavandchi, H., Roessner, S., Arabi, S., 2017. Quantifying groundwater exploitation induced subsidence in the Rafsanjan plain, southeastern Iran, using InSAR time-series and in situ measurements. Eng. Geol. 218, 134-151.

Pathak, D., Krahenbuhl, P., Donahue, J., Darrell, T., Efros, A. A., 2016. Context Encoders: Feature Learning by Inpainting. arXiv:1604.07379 [cs]. http://arxiv.org/abs/1604.07379. arXiv: 1604.07379 .
Redmon, J., Farhadi, A., 2016. YOLO9000: Better, Faster, Stronger. CoRR, abs/1612.08242. http://arxiv.org/abs/1612.08242.

Ronneberger, O., Fischer, P., Brox, T., 2015. U-Net: Convolutional Networks for Biomedical Image Segmentation. arXiv:1505.04597 [cs]. http://arxiv.org/abs/1505.04597. arXiv: 1505.04597.

Sensing, G. R., n.d. Software: GAMMA Remote Sensing AG.

Sentinel Data Access Annual Report 2017 - News - Sentinel Online, n.d.

Shamshiri, R., Motagh, M., Nahavandchi, H., Haghshenas Haghighi, M., Hoseini, M., 2020. Improving tropospheric corrections on large-scale Sentinel1 interferograms using a machine learning approach for integration with GNSS-derived zenith total delay (ZTD). Remote Sensing of Environment, 239, 111608. http://www.sciencedirect.com/science/article/pii/S0034425719 306285 .

Sharma, M., Verma, A., Vig, L., 2019. Learning to Clean: A GAN Perspective. arXiv:1901.11382 [cs]. http://arxiv.org/abs/1901.11382. arXiv: 1901.11382.

Simonyan, K., Zisserman, A., 2014. Very Deep Convolutional Networks for Large-Scale Image Recognition. CoRR, abs/1409.1556.

\section{SRTM 90m Digital Elevation Database v4.1, 2017.}

Wang, X., Gupta, A., 2016. Generative Image Modeling using Style and Structure Adversarial Networks. arXiv:1603.05631 [cs]. http://arxiv.org/abs/1603.05631. arXiv: 1603.05631.

Xu, J., Huang, Y., Liu, L., Zhu, F., Hou, X., Shao, L., 2019. Noisy-As-Clean: Learning Unsupervised Denoising from the Corrupted Image. CoRR, abs/1906.06878. http://arxiv.org/abs/1906.06878.

Yu, C., Li, Z., Penna, N. T., 2018a. Interferometric synthetic aperture radar atmospheric correction using a GPS-based iterative tropospheric decomposition model. Remote Sensing of Environment, 204, 109-121. http://www.sciencedirect.com/science/article/pii/S0034425717305011 .

Yu, C., Li, Z., Penna, N. T., Crippa, P., 2018b. Generic Atmospheric Correction Model for Interferometric Synthetic Aperture Radar Observations. Journal of Geophysical Research: Solid Earth, 123(10), 9202-9222. https://agupubs.onlinelibrary.wiley.com/doi/abs/10.1029/2017JB015305 .

Yu, C., Penna, N. T., Li, Z., 2017. Generation of real-time mode high-resolution water vapor fields from GPS observations. Journal of Geophysical Research: Atmospheres, 122(3), 2008-2025. https://agupubs.onlinelibrary.wiley.com/doi/abs/10.1002/2016JD025753.

Zebker, H. A., Rosen, P. A., Hensley, S., 1997. Atmospheric effects in interferometric synthetic aperture radar surface deformation and topographic maps. 